\title{
Cerebral protection Effect of Danhong Injection on CPB Rats
}

\author{
Dezhan $\mathrm{Li}^{1}$, Shuang $\mathrm{Han}^{2,}{ }^{*}$, Tao Xie ${ }^{1}$, Man $\mathrm{Li}^{3}$ \\ ${ }^{1}$ Department of Anesthesiology, Jingzhou Central Hospital, The Second Clinical Medical College, \\ Yangtze University, Jingzhou, Hubei 434020,China. \\ ${ }^{2}$ Department of Gynaecology, Jingzhou Central Hospital, The Second Clinical Medical College, \\ Yangtze University, Jingzhou, Hubei 434020,China. \\ ${ }^{3}$ Department of Oncology, Jingzhou Central Hospital, The Second Clinical Medical College, \\ Yangtze University, Jingzhou, Hubei 434020,China. \\ *Corresponding author: Shuang Han, E-mail:6939904@qq.com
}

Keywords: Danhong injection; CPB; rat; cerebral protection

\begin{abstract}
Objective: to observe the influence of Danhong injection on cerebral infarction area and neuron apoptosis rate of rats undergoing cardiopulmonary bypass (CPB) and then discuss cerebral protection effect of Danhong injection. Method: 24 healthy and adult SD rats were divided into sham operation group, CPB group and medicine intervention group at random. Each group includes 8 rats. Cerebral infarction area and neuron apoptosis rate of 3 groups were compared. Results: compared with CPB group, cerebral infarction area of medicine intervention group decreased $(\mathrm{P}<0.05)$. Compared with sham operation group, OD value of apoptotic cells in CPB group rose significantly $(\mathrm{P}<0.01)$. Compared with CPB group, OD value of apoptotic cells in medicine intervention group reduced obviously $(\mathrm{P}<0.01)$. Bax level and Caspase-3 level of CPB group were obviously higher than that of sham operation group, while Bcl-2 was significantly lower than that of sham operation group $(\mathrm{P}<0.01)$. Bax level and Caspase-3 level of medicine intervention group was significantly lower than that of CPB group, while Bcl-2 was significantly higher than that of $\mathrm{CPB}$ group $(\mathrm{P}<0.01)$. Conclusion: Danhong injection can play a good cerebral protection role through lowering cerebral infarction area and neuron apoptosis rate.
\end{abstract}

Medical research indicates that, continuous contact of heparinized blood with artificial surface wound tissue, and ischemia-reperfusion injury trigger pathophysiological reaction during CPB so that internal environment, tissues and organs have function disorder. This experiment aims to study the influence of Danhong injection on cerebral infarction area, neuron apoptosis rate and blood brain barrier damage caused by CPB rat ischemia so as to provide basis for cerebral protection effect of Danhong injection. 


\section{Material and Method}

\subsection{Material}

24 healthy and adult male clean-grade (SD) rates (provided by Animal Experiment Center of Tongji Medical College of HUST) were chosen, with the weight of (350-460)g. The animal experiment was executed in strict accordance with the experiment guide, and was approved by the Ethics Committee.

\subsection{Method}

\subsubsection{Animal model establishment and grouping}

The rats were divided into sham operation group, CPB group and medicine intervention group at radon. Each group includes 8 rats.

\subsubsection{CPB establishment}

Before the experiment, water ambrosia for $6 \mathrm{~h}$ was implemented for the rats, and $10 \%$ chloral hydrate $(300 \mathrm{mg} / \mathrm{kg}$, intraperitoneal injection) were used to narcotize the rats. Conventional fixation, mechanical ventilation and oxygen intaking were conducted. The rats were monitored with electrocardiogram lead.

The rats were cut longitudinally on the middle and right neck to make right carotid artery exposed. Then, they were fixed to prepare for CPB perfusion. Heparinization for the whole body was carried out. ACT reached (400-500)s for right atrium drainage. CPB loop adopted right jugular vein drainage and right carotid artery perfusion. $10 \mathrm{ml}$ injector was used to drain blood by gravity at $40 \mathrm{~cm}$ below the rat's heart. CPB priming fluid consisted of hydroxyethyl starch (12ml, 6\%), sodium bicarbonate $(2 \mathrm{ml}, 5 \%)$, and mannitol $(1 \mathrm{ml})$, with the total volume of $15 \mathrm{ml}$. After fixation, vena cava drainage tube and roller blood pump were opened for bypass. When the blood in the bleeding groove became fresh red arterial blood, the blood was pumped into the right carotid artery. The initial bypass flow was $(25-35) \mathrm{ml} /(\mathrm{kg} \cdot \mathrm{min})$, and later gradually rose to $(100-150) \mathrm{ml} /(\mathrm{kg} \cdot \mathrm{min})$. After stabilization, mechanical ventilation stopped, and the oxygenator was applied. Oxygen flow and perfusion volume kept (0.8-1.0):1. After bypass for $60 \mathrm{~min}$, mechanical ventilation is restored. Then, the perfusion volume decreased gradually, and machine was stopped. In the process of CPB establishment, heart rate, mean arterial pressure and other vital sign indexes were continuously monitored.

\subsubsection{Experimental method}

Sham operation group: cannula was conducted for each part, and mechanical ventilation for 60min was implemented, without bypass.

CPB group: equivalent normal saline was added in the priming fluid, and CPB was conducted for $60 \mathrm{~min}$.

Medicine intervention group: 2ml/kg Danhong injection (manufacturer: Buchang Pharmaceuticals; batch No.: GYZZ H110247) was added in the priming fluid, and CPB was conducted for $60 \mathrm{~min}$. 


\subsection{Detection index}

\subsubsection{Cerebral infarction area}

$100 \mathrm{~g} / \mathrm{L}$ chloral hydrate was used to narcotize the rats and then the rats were put to death. Their olfactory bulbs were removed. After the whole cerebrum was weighed and put in the refrigerator. After 20min, the cerebrum was taken out, and cut into $2 \mathrm{~mm}$-thick coronal plane brain slices from the frontal pole to polus occipitalis. They were kept out of the light and dyed for 30min. After dyeing, the normal tissue will present red, and ischemia tissue will present white ${ }^{[1]} .40 \mathrm{~g} / \mathrm{L}$ formaldehyde buffer solution was used to fix, and CAD image processing software was used to calculate brain slice area and white area of each slice. (cerebral infarction area/ whole cerebrum area) $\times 100 \%$ was used as the statistical index.

\subsubsection{Neuron apoptosis}

TUNEL method was applied to detect neuron apoptosis. 8 slices were chosen for each group. LOYMPUS BX51 microscope was used to choose 5 non-repeated views at random for camera shooting under $200 \mathrm{x}$ lens, and average integral optical density (OD, measured with Metamorph Dplo BX41 microscopic image analysis system) of apoptotic cells was measured. Meanwhile, Envision method was adopted to detect correlation factors of neuronal apoptosis (Bax, Caspase-3 and Bcl-2). The numerical proportion of sham operation group was transformed to 1 , and the numerical values of other groups were transformed by taking mean as the standard. The result was expressed with $\bar{X} \pm s^{[2]}$.

\subsection{Statistical method}

SPSS19.0 software was used to process experimental data. Statistical contrast was conducted with $\chi^{2}$ and t. $\mathrm{P}<0.01$ means the difference is significant.

\section{Result}

\subsection{Cerebral infarction area}

Compared with CPB group, cerebral infarction area of medicine intervention group decreased obviously, $\mathrm{P}<0.05$. The difference was significant. The details are shown in Tab.1.

Tab.1 Cerebral infarction area contrast of 3 groups

\begin{tabular}{lll}
\hline \multicolumn{1}{c}{ Group } & No. & Cerebral infarction area (\%) \\
\hline Sham operation group & 8 & 0 \\
CPB group & 8 & $35.01 \pm 4.18$ \\
Medicine intervention group & 8 & $20.1 \pm 1.02$ \\
$\mathrm{t}$ & $/$ & 9.801 \\
$\mathrm{P}$ & $/$ & $<0.01$ \\
\hline
\end{tabular}

\subsection{Neuronal apoptosis}

Neuronal apoptosis of CPB group increased obviously, while neuronal apoptosis of medicine intervention group declined significantly, as shown in Fig.1, Fig.2 and Fig.3. OD value of apoptotic 
cells in sham operation group was (3.65 \pm 0.33$)$; the value in CPB group was (14.60 \pm 0.80$)$. OD value of apoptotic cells in CPB group rose obviously, $\mathrm{t}=-35.789, \mathrm{P}<0.01$. The difference was significant.

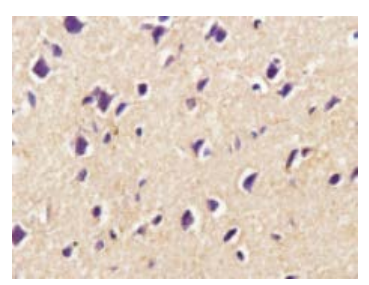

Fig.1 Sham operation group

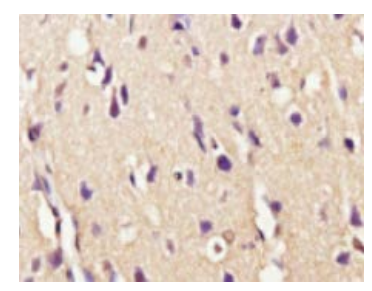

Fig.2 CPB group

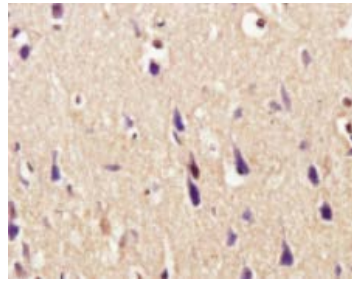

Fig.3 Medicine intervention group

Bax level and Caspase-3 level of CPB group were obviously higher than that of sham operation group, while Bcl-2 was obviously lower than that of sham operation group. Bax level and Caspase-3 level of medicine intervention group were obviously lower than that of CPB group, while Bcl-2 was obviously higher than $\mathrm{CPB}$ group, $\mathrm{P}<0.01$. The different was significant, as shown in Tab.2.

Tab.2 Correlation factors of neuronal apoptosis in 3 groups $(\bar{X} \pm s)$

\begin{tabular}{lllll}
\hline Group & Number of samples & Bax & Caspase-3 & Bcl-2 \\
\hline $\begin{array}{l}\text { Sham operation } \\
\text { group }\end{array}$ & 8 & $1 \pm 0.12$ & $1 \pm 0.07$ & $1 \pm 0.30$ \\
$\begin{array}{l}\text { CPB group } \\
\text { Medicine }\end{array}$ & 8 & $9.10 \pm 3.40$ & $21.46 \pm 8.30$ & $0.20 \pm 0.01$ \\
$\begin{array}{l}\text { intervention } \\
\text { group }\end{array}$ & 8 & $1.41 \pm 0.30$ & $4.55 \pm 1.40$ & $3.22 \pm 1.10$ \\
t1 & $/$ & -6.734 & -6.972 & 7.538 \\
P1 & $/$ & $<0.01$ & $<0.01$ & $<0.01$ \\
t2 & $/$ & -6.372 & -5.682 & 7.750 \\
P2 & $/$ & $<0.01$ & $<0.01$ & $<0.01$ \\
\hline
\end{tabular}

Note: $\mathrm{t} 1$ and P1 represent comparison of sham operation group and CPB group; t2 and P2 represent comparison of CPB group and medicine intervention group.

\section{Discussion}

In this experiment, CPB animal experiment was chosen as the object to discuss cerebral protection effect and mechanism of Danhong injection. Danhong injection is a common traditional Chinese medicinal material to assist treatment of cerebral infarction. Its main ingredients are salvia miltiorrhiza and safflower which have the good effect of inhibiting cell apoptosis, scavenging free radical and improving microcirculation ${ }^{[3]}$.

Cerebral infarction area is an important index to evaluate cerebral ischemia-reperfusion injury ${ }^{[4]}$. Seeing from the experimental result, cerebral infarction in the medicine intervention group for which Danhong injection was applied significantly declined, compared with CPB group. This conclusion is consistent with Chen Gang's report. This fully verifies the success in model establishment ${ }^{[5]}$. Cell apoptosis is one kind of "programmed cell death". In other words, it is active "suicide" behavior of cells ${ }^{[6]}$. The typical features of cell apoptosis include chromatin agglutination and cell nucleus collapse. Thus, if the above circumstances are found, cell apoptosis likely exists. TUNEL method was used to observe neurons of rats. It was found that, after dyeing, neuronal apoptosis of CPB group rose obviously, while neuronal apoptosis of medicine intervention group 
declined significantly, compared with CPB group. At the same time, OD detection showed that OD value of apoptotic cells in CPB group rose obviously $(\mathrm{P}<0.01)$. Medicine intervention group declined significantly, compared with $\mathrm{CPB}$ group $(\mathrm{P}<0.01)$. This indicates that neuronal apoptosis is one of causes for CPB cerebral injury.

In this experiment, the relationship between Danhong injection and neuronal apoptosis was judged through measuring expression degree of apoptosis-related factors. According to the data, neuron as a basic functional unit of neural system has the same gene with common cells. Apoptosis may happen to neurons after they are activated by apoptosis signal ${ }^{[7]}$. The most classical apoptosis-related factors are Caspase family and Bcl-2 gene family ${ }^{[8]}$. Bax (induce apoptosis), Caspase-3 (apoptosis accelerant) and Bcl-2 (anti-apoptosis) are almost related to apoptosis. They are the most classical apoptosis factors. According to Tab.2, Danhong injection can effectively inhibit the expression of Bax and Caspase-3, and promote Bcl-2 expression. This verifies Danhong injection can reduce apoptosis rate.

In conclusion, Danhong injection can play a good cerebral protection role through reducing cerebral infarction area and neuronal apoptosis rate.

\section{References}

[1] KJ Gon,SD Jun,K Hyun-Ji,et al.Impaired Inactivation of L-Type Ca2+Current as a Potential Mechanism for Variable Arrhythmogenic Liability of HERG K+Channel Blocking Drugs:[J].Plos One, 2016,11 (3) :e0149198.

[2] EY Suh,MZ Yin,H Lin,et al. Maxi-K channel (BKCa) activity veils the myogenic tone of mesenteric artery in rats.[J].Physiological Reports,2017,5 (14) :e13330.

[3] T Matsumoto,T Szasz,RC Tostes, et al.Impaired $\beta$-adrenoceptor-induced relaxation in small mesenteric arteries from DOCA-salt hypertensive rats is due to reduced $K(\mathrm{Ca})$ channel activity.[J].Pharmacological Research,2012,65(5):537-545

[4] H Fan,M Li,L Yu,et al.Effects of Danhong Injection on platelet aggregation in hyperlipidemia rats[J].Journal of Ethnopharmacology,2017,212:67-73.

[5] J Wan,H Wan,R Yang,et al.Protective effect of Danhong Injection combined with Naoxintong Capsule on cerebral ischemia-reperfusion injury in rats. [J].Journal of Ethnopharmacology,2017,211:348-357.

[6] Huang Hai-feng.Guo Fei,Cao Yuan-zhao,et al.Neuroprotec-tion by manganese supero-xide dismutase (MnSOD) mimics:an-tioxidant effect and oxidative stress regulation in acute experimen-tal stroke[J]. CNS Neurosci Therap, 2012, 10(18) : 811.

[7] L Dong,J Zheng, C Liu,et al.Detection of serum cardiac biomarkers under moderate and high level of $+G_{-} Z$ exposure[J].Medical Journal of Air Force, 2017(03):145-148.

[8] Lan R,Zhang Y,Xiang J,et al.Xiao-Xu-Ming decoction pre-serves mitochondrial integrity and reduces apoptosis after focal cerebral ischemia and reperfusion via the mitochondrial p53 path-way [J]. J Ethnopharmacol, 2014, 151(1): 307. 